dementia. Although real sadness is avoided, sad music is often listened to keenly. In a seminal work, it was reported that listening to sad music led to four different types of reward, relating to imagination, emotion regulation, empathy and lack of 'real-life' implications (Taruffi \& Koelsch, 2014).

\section{Conclusion and future directions}

The present article provides only a bird's eye view of the vast subject, which has grown exponentially in the recent past and is continuing to do so. Musical training and evidence-based music therapy improve sensorimotor, language and cognitive functions in the non-musical domain. So far, the Western classical music tradition has been scientifically examined to a far greater extent than music from any other tradition. The findings are generalisable, however, as different forms of music from various cultures have certain common features. Nonetheless, traditional music of various cultures should be systematically studied to determine their unique value, if any, in a therapeutic scenario. Scientific investigations of the innumerable ragas of ICM are still in their infancy. Systematic studies on various psychiatric conditions are imperative.

\section{References}

Biswas, A., Hegde, S., Jhunjhunwala, K., et al (2016) Two sides of the same coin: impairment in perception of temporal components of rhythm and cognitive functions in Parkinson's disease. Basal Ganglia, 6, 63-70.
Bruscia, K. (2000) Definindo Musicoterapia [Defining Music Therapy]. Enelivros.

Chanda, M. L. \& Levitin, D. J. (2013) The neurochemistry of music Trends in Cognitive Sciences, 17, 179-193.

Chandrashekaran, N. (2015) Musical Deficits in Schizophrenia - Its Relation with Deficits in Cognition and Emotion Recognition. MPhil thesis in clinical psychology, National Institute of Mental Health and Neurosciences, Bangalore, India

Gupta, U. \& Gupta, B. S. (2016) Gender differences in psychophysiological responses to music listening. Music and Medicine, 8, 53-64

Hegde, S. (2014) Music-based cognitive remediation therapy for patients with traumatic brain injury. Frontiers in Neurology, 5, 34

Hegde, S., Aucouturier, J.-J., Ramanujam, B., et al (2012) Variations in emotional experience during phases of elaboration of north Indian raga performance. In 12th International Conference on Music Perception and Cognition and the 8th Triennial Conference of the Cognitive Sciences of Music, July 23-28 2012 (eds E. Cambourpoulos, et al), pp. 412-413.

Hegde, S., Bharath, R. D., Rao, M. B., et al (2016) Preservation of cognitive and musical abilities of a musician following surgery for chronic drug-resistant temporal lobe epilepsy: a case report. Neurocase, 22, 1-6.

Jairazbhoy, N. A. (1995) The Ragas of North Indian Music: Their Structure and Evolution. Popular Prakashan Pvt Ltd.

Maratos, A., Gold, C., Wang, X., et al (2008) Music Therapy for Depression. Cochrane Library.

Mossler, K., Chen, X., Heldal, T. O., et al (2011) Music therapy for people with schizophrenia and schizophrenia-like disorders. Cochrane Database of Systematic Reviews, (2), Cd004025.

Taruffi, L. \& Koelsch, S. (2014) The paradox of music-evoked sadness: an online survey. Plos One, 9, E110490.

Zatorre, R. (2005) Music, the food of neuroscience? Nature, 434 312-315.

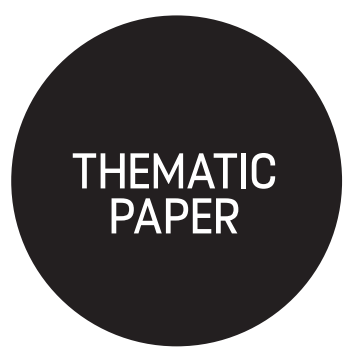

\title{
Music performance anxiety in classical musicians - what we know about what works
}

\author{
Raluca Matei ${ }^{1}$ and Jane Ginsborg ${ }^{2}$
}

'Royal Northern College of Music, Manchester, UK, email raluca. matei@student rncm.ac.uk

2Royal Northern College of Music Manchester, UK, email jane. ginsborg@rncm.ac.uk
When pursued professionally, the demands of musical training and performance can interfere with musicians' well-being and health. Music performance anxiety, while energising at optimal levels, impairs performance quality when excessive. A range of interventions has been explored to address it. However, the poor methodological quality of such studies and the complexity of this issue should mobilise further research resources in this direction.

\section{Music performance anxiety}

Might the mere presence of an audience be enough to turn music making, so often therapeutic, into an anxiety-enhancing activity? Fancourt et al (2015) found higher levels of glucocorticoids (cortisol and cortisone) in singers performing in public than when they were singing without an audience. Furthermore, research using a virtual reality performance simulator demonstrated that the audience does not even have to be 'real' for anxiety to be triggered or heart rate raised (Aufegger et $a l, 2016)$.

Those who succeed in entering the highly competitive field of classical music must not only possess personal attributes such as determination and resilience but also acquire cognitive and social skills, instrument-specific motor skills, coping skills adequate to the psychological demands of public performance, and the ability to manage their time and be responsible for their physical and mental health. While musicians report the highest level of job satisfaction, they are also among the five occupational groups most likely to report mental illness (Brodsky, 1996). 
Performance anxiety has been investigated in a variety of contexts, including test-taking, public speaking, writing, sexual performance, sport and the performing arts (dance, music and acting). Music performance anxiety (MPA) is a complex phenomenon caused by the interaction of many factors, including genetics, environmental stimuli and the individual's experience, emotions, cognitions and behaviours (Kenny, 2011). It manifests via three elements, independent to varying extents: cognitions, autonomic arousal and behaviours (Kenny, 2005). While a certain degree of performance anxiety is facilitative and normal, it can sometimes become debilitating and even qualify as a mental disorder. According to DSM-5 (American Psychiatric Association, 2013), performance anxiety is a subtype of social anxiety disorder (SAD). In order for someone to qualify as having SAD, they need to have suffered from persistent fear, anxiety or avoidance for at least 6 months, and to have considerably impaired social, occupational or general functioning. Although there are correlations between MPA and certain aspects of SAD, such as fear of negative evaluations and the perceived exaggerated consequences of such evaluations, particularly in solo performance, their interactions remain unclear (Goren, 2014).

According to the Yerkes-Dodson law, optimal performance is associated with a moderate level of arousal. A more nuanced extension of this law has been proposed, however, identifying three sources of stress that interact differently in individuals: trait anxiety (a personality characteristic), situational stress (in public performance, auditions, etc.) and task mastery (in the context of both undemanding, rehearsed material and complex, largely unknown works) (Wilson \& Roland, 2002). Thus, performers' anxiety is likely to be reduced as they engage in task mastery, transforming a difficult work into one that is both more familiar and easier to play.

We know that memory can be affected by anxiety. Sport psychologists and researchers studying test anxiety have proposed two attentional processes to explain 'choking' (performance impairment caused by excessive psychological pressure): distraction and explicit monitoring. Distraction depletes attentional resources as working memory is occupied by interfering worries and the individual is unable to focus on the task at hand. Conversely, monitoring can have a counterproductive effect because it disrupts the performance of a well practised skill that would otherwise be automatic. Still, it remains unclear whether such distinctions are clear enough, whether they can meaningfully inform different interventions and whether choking and underperformance are conceptually distinguishable (Mesagno et al, 2015).

According to a survey of more than 2000 professional musicians conducted by the International Conference of Symphony and Opera Musicians (ICSOM), the largest sample to date, $24 \%$ suffered from stage fright, $13 \%$ reported acute anxiety and 17\% reported depression (Fishbein et al, 1988). The highest levels of MPA are generally triggered by solo performances (Spahn et al, 2016), while orchestral players rated auditions as the most likely to produce MPA and practising alone the least. Musicians playing in pit orchestras (e.g. in opera, ballet and theatre productions) experience more severe MPA than musicians who combine playing in pit orchestras with performing on stage (Kenny et al, 2016).

The three causes of MPA most commonly cited by musicians are 'pressure from self', 'excessive arousal' and 'inadequate preparation for performance' (Kenny et al, 2014). Orchestral players might well find inadequate preparation a general and persistent stressor, since they tour and perform extensively with too little time to rehearse and digest the repertoire, often relying on their ability to sight-read. Coping strategies reported by musicians include increasing practice, recommended by $91 \%$ of respondents to Kenny et al's 2014 survey; deep breathing; positive self-talk; mock performance practice; familiarising themselves with the performance venue; relaxation methods; discussions; and the use of medication.

Of the musicians from American orchestras who responded to the ICSOM survey (Fishbein et al, 1988), 27\% took propranolol or another beta-blocker, most of them without a doctor's prescription, and $96 \%$ of these respondents reported these effective in reducing MPA. Of the Australian musicians who responded to Kenny et al's (2014) survey, $31 \%$ reported taking beta-blockers to alleviate MPA, while $12 \%$ used alcohol, $5 \%$ anxiolytics and $4 \%$ antidepressants. Recent data from 1500 Norwegian musicians suggest higher use of psychotropic medication (e.g. sedatives, antidepressants, hypnotics and medication for attention-deficit hyperactivity disorder), especially among string players and particularly when compared with managers and technicians. Similarly, musicians were three times more likely to use psychotherapy than the general workforce (Vaag et al, 2016).

\section{Treatment and prevention}

Three systematic reviews of interventions aimed to reduce MPA (McGinnis \& Milling, 2005; Kenny, 2005; Brugués, 2011) and an unpublished metaanalysis (Goren, 2014) have been conducted to date. McGinnis \& Milling (2005) included nine studies and suggested that the most effective treatments incorporate cognitive restructuring and exposure therapy. The other two incorporated the same 21 studies, with Brugués (2005) reviewing an additional five and concluding that cognitive-behavioural therapy (CBT) might be particularly effective. Brugués also concluded that, although the use of beta-blockers might reduce some physiological manifestations of MPA, they also increase salivation. As they can thereby interfere with singers' and wind players' performance, Brugués recommended that beta-blockers be used cautiously, generally alongside psychological techniques. Goren (2014) conducted a meta-analysis of 29 studies and concluded that four therapies (behavioural, complementary and alternative, 
cognitive, and combined) were moderately effective, with a mean effect size of 0.64. Most effective was a combination of two or more types of therapy.

More recent research has explored the effectiveness of multimodal interventions. Spahn et al (2016) evaluated the combination of behavioural exposure (via mock orchestral auditions and recorded performances) with group discussion, expert feedback and cognitive strategies; Steyn $e t$ al (2016) added mindfulness training to an array of cognitive-behavioural elements. In both cases interventions produced lower scores for both MPA and state anxiety. Virtual reality exposure training also reduced performance anxiety and improved performance quality, especially among anxietyprone musicians (Bissonnette et al, 2015).

Juncos \& Markman (2015) were the first to report an investigation of the effects of acceptance and commitment therapy (ACT). An undergraduate violinist with debilitating MPA showed both clinically significant improvements in MPA and better performance quality after ten sessions.

Psychological skills training (e.g. goal setting, concentration, imagery, self-talk, arousal regulation) has been used successfully in sports for the last five decades, but rarely with musicians (Hatfield, 2016). Some data on the thoughts of musicians under pressure suggest that unproductive coping strategies might be positively correlated with both MPA (Thomas \& Nettlebeck, 2014) and performance quality. The relationship between performance quality and performance anxiety is indeed complex and there might be scope for studying them separately. However, it is difficult to assess performance quality other than subjectively. Furthermore, although reframing performance anxiety as excitement might not reduce levels of anxiety (Brooks, 2014), performance quality might nevertheless improve.

\section{Conclusions}

Although many interventions aimed at reducing or preventing MPA in both professional musicians and music students have been implemented and evaluated, it is difficult to draw firm conclusions since so many reported studies are methodologically weak; limitations acknowledged by their authors include design, participants, intervention characteristics and outcome measures. Several studies were conducted over short periods with small samples and/or no control group. Given that anxiety can also be enhancing and facilitating, and that compliance is likely to influence therapeutic outcomes, it is perhaps surprising that these are so rarely considered in research. Although it is hard to isolate active ingredients in complex interventions, these - particularly when they involve cognitive-behavioural elements - hold more promise in terms of managing MPA. More rigorous investigations, however, are warranted.

\section{References}

American Psychiatric Association (2013) Diagnostic and Statistical Manual of Mental Disorders (5th edn) (DSM-5). American Psychiatric Publishing.
Aufegger, L., Perkins, R., Wasley, D., et al (2016) Musicians' perceptions and experiences of using simulation training to develop performance skills. Psychology of Music, advance online publication. https://doi.org/10.1177/0305735616666940.

Bissonnette, J., Dube, F., Provencher, M. D., et al (2015) Virtual reality exposure training for musicians: its effect on performance anxiety and quality. Medical Problems of Performing Artists, 30 , 169-177

Brodsky, W. (1996) Music performance anxiety reconceptualised: a critique of current research practices and findings. Medical Problems of Performing Artists, 3, 88-98.

Brooks, A. W. (2014) Get excited: reappraising pre-performance anxiety as excitement. Journal of Experimental Psychology: General, 143, 1144-1158.

Brugués, A. O. (2011) Music performance anxiety - part 2. A review of treatment options. Medical Problems of Performing Artists, 26 164-171.

Fancourt, D. Aufegger, L. \& Williamon, A. (2015) Low-stress and high-stress singing have contrasting effects on glucocorticoid response. Frontiers in Psychology, 6. https://doi.org/10.3389/ fpsyg.2015.01242

Fishbein, M., Middlestadt, S. E., Ottati, V., et al (1988) Medical problems among ICSOM musicians. Medical Problems of Performing Artists, 3, 1-8.

Goren, L. (2014) A Meta-analysis of Nonpharmacological Psychotherapies for Music Performance Anxiety. Unpublished doctoral dissertation, California Institute of Integral Studies, San Francisco, California, USA

Hatfield, J. L. (2016) Performing at the top of one's musical game. Frontiers in Psychology, 7. https://doi.org/10.3389/ fpsyg.2016.01356.

Juncos, D. G. \& Markman, E. J. (2015) Acceptance and commitment therapy for the treatment of music performance anxiety: a single subject design with a university student. Psychology of Music, advance online publication. https://doi org/10.1177/0305735615596236.

Kenny, D. T. (2005) A systematic review of treatments for music performance anxiety. Anxiety, Stress, and Coping: An International Journal, 18, 183-208. https://doi. org/10.1080/10615800500167258.

Kenny, D. T. (2011) The Psychology of Music Performance Anxiety. Oxford University Press.

Kenny, D. T., Driscoll, T. \& Ackermann, B. (2014) Psychological wellbeing in professional orchestral musicians in Australia: a descriptive population study. Psychology of Music, 2, 210-232.

Kenny, D. T., Driscoll, T. R. \& Ackermann, B. (2016) Is playing in the pit really the pits? Pain, strength, music performance anxiety, and workplace satisfaction in professional musicians in stage, pit, and combined stage/pit orchestras. Medical Problems of Performing Artists, 31, 1-7.

McGinnis, A. M. \& Milling, L. S. (2005) Psychological treatment of musical performance anxiety: current status and future directions. Psychotherapy: Theory, Research Practice, Training, 42, 357-373.

Mesagno, C., Geukes, K. \& Larkin, P. (2015) Choking under pressure: a review of current debates, literature, and interventions. In Contemporary Advances in Sport Psychology: A Review (eds S. Mellalieu \& S. Hanton), pp. 148-174. Routledge.

Spahn, C. Walther, J.-C. \& Nusseck, M. (2016) The effectiveness of a multimodal concept of audition training for music students in coping with music performance anxiety. Psychology of Music, 4 , 893-909

Steyn, B. J. M., Steyn, M. H., Maree, D. J. F., et al (2016) Psychological skills and mindfulness training effects on the psychological wellbeing of undergraduate music students: an exploratory study. Journal of Psychology in Africa, 26, 167-171.

Thomas, J. P. \& Nettelbeck, T. (2014) Performance anxiety in adolescent musicians. Psychology of Music, 42, 624-634.

Vaag, J., Bjorngaard, J. H. \& Bjekeset, O. (2016) Use of psychotherapy and psychotropic medication among Norwegian musicians compared to the general workforce. Psychology of Music, advance online publication. https://doi. org/10.1177/0305735616637132.

Wilson, G. D. \& Roland, D. (2002) Performance anxiety. In The Science and Psychology of Music Performance: Creative Strategies for Teaching and Learning (eds R. Parncutt \& G. E. McPherson), pp. 47-61. Oxford University Press. 